Malignes Melanom

\title{
Immunonkologische Kombination floppt in Phase-III-Studie
}

Um die Effektivität der immunonkologischen Therapie weiter zu steigern, werden heute Kombinationen von PD-(L)-1-Inhibitoren mit unterschiedlichen Substanzen intensiv geprüft. Allerdings nicht immer mit dem erwünschten Ergebnis: In der Phase-IIIStudie ECHO-301 beim inoperablen Melanom war das Regime mit Pembrolizumab und dem IDO-1-Inhibitor Epacadostat nicht effektiver als Pembrolizumab mono.

Das Enzym IDO-1 (Indoleamin-2,3-dioxygenase 1) katalysiert den ersten Schritt beim Abbau von Tryptophan im Kynurenin-Weg. Die Abnahme dieser Aminosäure und der gleichzeitige Anstieg von Kynurenin und weiteren Metaboliten im Tumorgewebe führt im Mikromilieu des Tumors zu einem Shift in Richtung Immunsuppression. Damit kann der Tumor der Immunüberwachung besser entgehen, erläuterte Prof. Georgina V. Long, Sydney/Australien.

Mit Epacadostat wurde ein potenter IDO1-Blocker entwickelt, der als kompetitiver Inhibitor von Tryptophan wirkt. In Kombination mit einem PD-1-Inhibitor wie Pembrolizumab sollte die Immuntoleranz von Tumoren noch effektiver als unter alleiniger CheckpointBlockade ausgeschaltet werden. In der PhaseI/II-Studie ECHO-202/KEYNOTE-037 erwies sich dieses Regime bei Patienten mit fortgeschrittenem Melanom mit einer Gesamtansprechrate von $55 \%$ und einem progressionsfreien Überleben (PFS) von 22,8 Monaten als erfolgreich (1).

Allerdings wurden diese positiven Ergebnisse in der Studie ECHO-301/KEYNOTE-252 nicht bestätigt (2). Die Phase-III-Studie schloss 701 Patienten mit inoperablem Melanom (Stadium III/IV) ein, die randomisiert mit Pembroli-
zumab/Epacadostat oder der PembrolizumabMonotherapie behandelt wurden. Beim primären Endpunkt PFS schnitten beide Arme mit median 4,7 bzw. 4,9 Monaten nahezu identisch ab. Auch konnte in Subgruppenanalysen kein Kollektiv identifiziert werden, das stärker von der Kombination profitierte. Beim Gesamtüberleben (OS) zeichnet sich ebenfalls kein Vorteil der Kombination ab: Zwar ist der Median in beiden Armen noch nicht erreicht; die Ereigniskurven verlaufen jedoch praktisch deckungsgleich. Die Responseraten sind mit $34,2 \%$ im Kombinations- und 31,5\% im Mono-Arm ebenfalls ähnlich. Das externe Data Monitoring Committee hat sich daher mittlerweile für den Abbruch der Studie ausgesprochen.

$$
\text { Dr. Ine Schmale, Westerburg }
$$

\section{Literatur}

1. Hamid 0 et al. ESMO 2017, Abstract 12140. 2. Long GV et al. ASCO 2017, Abstract 108.

Quelle: 54. Jahrestagung der American Society of Clinical Oncology (ASCO) vom 1. bis 5. Juni 2018 in Chicago/USA. 